\title{
Plain strain problem of poroelasticity using eigenvalue approach
}

\author{
RAJneEsh KUMAR ${ }^{1}$, ASEem Miglani ${ }^{2}$ and N R GARG ${ }^{3}$ \\ ${ }^{1}$ Department of Mathematics, Kurukshetra University, Kurukshetra 136119, Haryana, India. \\ ${ }^{2}$ Department of Mathematics, M. M. Engineering College, Mullana 133 203, Ambala, Haryana, India. \\ ${ }^{3}$ Department of Mathematics, Maharshi Dayanand University, Rohtak 124001, Haryana, India.
}

A plain strain problem of an isotropic elastic liquid-saturated porous medium in poroelasticity has been studied. The eigenvalue approach using the Laplace and Fourier transforms has been employed and these transforms have been inverted by using a numerical technique. An application of infinite space with concentrated force at the origin has been presented to illustrate the utility of the approach. The displacement and stress components in the physical domain are obtained numerically. The results are shown graphically and can be used for a broad class of problems related to liquid-saturated porous media.

\section{Introduction}

Liquid-saturated porous solids are often present on and below the surface of the Earth. Cramplin (1987) has explained that the fluid in the pores plays an important role in the occurrence of earthquakes. Due to this, as well as the significance of liquid-saturated porous media in many engineering problems, the liquid-saturated porous media are of great concern and have many applications in various fields such as earthquake engineering, seismology, geomechanics etc. Biot $(1956 \mathrm{a}, \mathrm{b})$ developed the theory of wave propagation in fluid-saturated porous materials. Deresiewicz and Skalak (1963) derived the boundary conditions appropriate for the continuity requirements at the interface of such materials. Most of the poroelastic problems solved so far, involve the use of potential functions. However, the use of the eigenvalue approach has the advantage of finding the solutions of equations in the coupled form directly, in the matrix notations, whereas the potential function approach requires decoupling of equations. Yet, much work has not been done in liquid-saturated porous media using the eigenvalue approach.

In this paper, we apply the eigenvalue approach following Laplace and Fourier transformations to the general plain strain problem of a homogeneous, iso- tropic, elastic liquid-saturated porous medium with reference to the theory developed by Biot. The solutions are obtained in the transformed domain and are inverted by using a numerical technique.

\section{Basic equations}

The field equations for a homogeneous, isotropic, elastic liquid-saturated porous medium are given by Biot (1956a,b) as

$$
\begin{aligned}
& N \nabla^{2} \vec{u}+\operatorname{grad}\{(D+N) e+Q \varepsilon\} \\
& =\frac{\partial^{2}}{\partial t^{2}}\left\{\rho_{11} \vec{u}+\rho_{12} \vec{U}\right\}+b \frac{\partial}{\partial t}(\vec{u}-\vec{U}), \\
& \operatorname{grad}\{Q e+R \varepsilon\}=\frac{\partial^{2}}{\partial t^{2}}\left\{\rho_{12} \vec{u}+\rho_{22} \vec{U}\right\} \\
& \quad-b \frac{\partial}{\partial t}(\vec{u}-\vec{U}),
\end{aligned}
$$

where $\vec{u}$ and $\vec{U}$ are the displacements in the solid and liquid parts of the porous aggregate respectively; $e=\operatorname{div} \vec{u}$ and $\varepsilon=\operatorname{div} \vec{U}$ are the corresponding dilatations; $D, N, Q$, and $R$ are the elastic constants for the solid-liquid aggregate, $D$ and $N$ correspond to the Lame modulii of the material, $Q$ is a measure of coupling between the volume change of solid and

Keywords. Plain strain; liquid-saturated porous; eigenvalue; Laplace and Fourier transforms. 
liquid, and $R$ is the pressure that must be exerted on the liquid to force a given volume of it into the porous aggregate while the total volume remains the same; $\rho_{11}, \rho_{12}$ and $\rho_{22}$ are the dynamical coefficients, where $\rho_{12}$ represents the mass coupling parameter between the fluid and solid; $b$ is the dissipation coefficient.

The stresses in the solid $\sigma_{i j}(i, j=x, y, z)$ and liquid $\sigma$ are given by

$$
\sigma_{i j}=(D e+Q \varepsilon) \delta_{i j}+2 N e_{i j}, \quad \sigma=Q e+R \varepsilon,
$$

where $\delta_{i j}$ is the Kronecker delta and

$$
e_{i j}=\frac{1}{2}\left(\frac{\partial u_{i}}{\partial x_{j}}+\frac{\partial u_{j}}{\partial x_{i}}\right) \text {. }
$$

\section{Formulation and solution}

We are considering a two-dimensional plain strain problem with

$$
\vec{u}=(u, 0, w), \quad \vec{U}=(U, 0, W),
$$

therefore, the field equations (1) and (2) in the components form reduce to

$$
\begin{aligned}
& N\left(\frac{\partial^{2} u}{\partial x^{2}}+\frac{\partial^{2} u}{\partial z^{2}}\right)+(D+N)\left(\frac{\partial^{2} u}{\partial x^{2}}+\frac{\partial^{2} w}{\partial x \partial z}\right) \\
& \quad+Q\left(\frac{\partial^{2} U}{\partial x^{2}}+\frac{\partial^{2} W}{\partial x \partial z}\right)=\rho_{11} \ddot{u}+\rho_{12} \ddot{U}+b(\dot{u}-\dot{U}), \\
& N\left(\frac{\partial^{2} w}{\partial x^{2}}+\frac{\partial^{2} w}{\partial z^{2}}\right)+(D+N)\left(\frac{\partial^{2} u}{\partial x \partial z}+\frac{\partial^{2} w}{\partial z^{2}}\right) \\
& \quad+Q\left(\frac{\partial^{2} U}{\partial x \partial z}+\frac{\partial^{2} W}{\partial z^{2}}\right)=\rho_{11} \ddot{w}+\rho_{12} \ddot{W}+b(\dot{w}-\dot{W}), \\
& Q\left(\frac{\partial^{2} u}{\partial x^{2}}+\frac{\partial^{2} w}{\partial x \partial z}\right)+R\left(\frac{\partial^{2} U}{\partial x^{2}}+\frac{\partial^{2} W}{\partial x \partial z}\right) \\
& \quad=\rho_{12} \ddot{u}+\rho_{22} \ddot{U}-b(\dot{u}-\dot{U}), \\
& Q\left(\frac{\partial^{2} u}{\partial x \partial z}+\frac{\partial^{2} w}{\partial z^{2}}\right)+R\left(\frac{\partial^{2} U}{\partial x \partial z}+\frac{\partial^{2} W}{\partial z^{2}}\right) \\
& \quad=\rho_{12} \ddot{w}+\rho_{22} \ddot{W}-b(\dot{w}-\dot{W}),
\end{aligned}
$$

where the superposed dots represent time differentiation.

We define the following non-dimensional quantities as

$$
\begin{aligned}
x^{\prime} & =\frac{\omega x}{\alpha_{2}}, \quad z^{\prime}=\frac{\omega z}{\alpha_{2}}, \quad t^{\prime}=\omega t, \\
u^{\prime} & =\frac{\rho \omega \alpha_{2} u}{H}, \quad w^{\prime}=\frac{\rho \omega \alpha_{2} w}{H}, \quad U^{\prime}=\frac{\rho \omega \alpha_{2} U}{H}, \\
W^{\prime} & =\frac{\rho \omega \alpha_{2} W}{H}, \\
\sigma_{z z}^{\prime} & =\frac{\sigma_{z z}}{P}, \quad \sigma_{x z}^{\prime}=\frac{\sigma_{x z}}{P}, \quad \sigma^{\prime}=\frac{\sigma}{P},
\end{aligned}
$$

where

$$
p=D+2 N, \quad \rho=\rho_{11}+2 \rho_{12}+\rho_{22}, \quad H=P+2 Q+R,
$$

$\omega$ is the angular frequency and $\alpha_{2}$, the velocity of slow dilatational wave in the liquid-saturated porous medium. Using these quantities (6) in equations (5) and after suppressing the dashes, we obtain

$$
\begin{aligned}
& \frac{\partial^{2} u}{\partial x^{2}}+a^{2} \frac{\partial^{2} u}{\partial z^{2}}+\left(1-a^{2}\right) \frac{\partial^{2} w}{\partial x \partial z}+b^{2}\left(\frac{\partial^{2} U}{\partial x^{2}}+\frac{\partial^{2} W}{\partial x \partial z}\right) \\
& \quad=c^{2}\left\{\left(R_{11} \ddot{u}+R_{12} \ddot{U}\right)+f^{2}(\dot{u}-\dot{U})\right\} \\
& a^{2} \frac{\partial^{2} w}{\partial x^{2}}+\frac{\partial^{2} w}{\partial z^{2}}+\left(1-a^{2}\right) \frac{\partial^{2} u}{\partial x \partial z}+b^{2}\left(\frac{\partial^{2} U}{\partial x \partial z}+\frac{\partial^{2} W}{\partial z^{2}}\right) \\
& \quad=c^{2}\left\{\left(R_{11} \ddot{w}+R_{12} \ddot{W}\right)+f^{2}(\dot{w}-\dot{W})\right\} \\
& b^{2}\left(\frac{\partial^{2} u}{\partial x^{2}}+\frac{\partial^{2} w}{\partial x \partial z}\right)+d^{2}\left(\frac{\partial^{2} U}{\partial x^{2}}+\frac{\partial^{2} W}{\partial x \partial z}\right) \\
& \quad=c^{2}\left\{\left(R_{12} \ddot{u}+R_{22} \ddot{U}\right)-f^{2}(\dot{u}-\dot{U})\right\} \\
& b^{2}\left(\frac{\partial^{2} u}{\partial x \partial z}+\frac{\partial^{2} w}{\partial z^{2}}\right)+d^{2}\left(\frac{\partial^{2} U}{\partial x \partial z}+\frac{\partial^{2} W}{\partial z^{2}}\right) \\
& \quad=c^{2}\left\{\left(R_{12} \ddot{w}+R_{22} \ddot{W}\right)-f^{2}(\dot{w}-\dot{W})\right\}
\end{aligned}
$$

where

$$
\begin{aligned}
a^{2} & =\frac{N}{D+2 N}, \quad b^{2}=\frac{Q}{D+2 N}, \quad c^{2}=\frac{\alpha_{2}^{2} \rho}{D+2 N}, \\
d^{2} & =\frac{R}{D+2 N}, \quad f^{2}=\frac{b}{\rho \omega}, \\
R_{11} & =\frac{\rho_{11}}{\rho}, \quad R_{12}=\frac{\rho_{12}}{\rho}, \quad R_{22}=\frac{\rho_{22}}{\rho} .
\end{aligned}
$$

Applying the Laplace transformation with respect to $t$

$$
\begin{array}{r}
\{\bar{u}(x, z, p), \bar{w}(x, z, p), \bar{U}(x, z, p), \bar{W}(x, z, p)\} \\
=\int_{0}^{\infty}\{u(x, z, t), w(x, z, t), U(x, z, t), \\
W(x, z, t)\} e^{-p t} \mathrm{~d} t,
\end{array}
$$

and then the Fourier transformation with respect to $x$

$$
\begin{array}{r}
\{\hat{u}(q, z, p), \hat{w}(q, z, p), \hat{U}(q, z, p), \hat{W}(q, z, p)\} \\
=\frac{1}{2 \pi} \int_{-\infty}^{\infty}\{\bar{u}(x, z, p), \bar{w}(x, z, p), \bar{U}(x, z, p), \\
\bar{W}(x, z, p)\} e^{i q x} \mathrm{~d} x,
\end{array}
$$

on equations (7), we obtain a system of equations, which can be written as

$$
A V^{\prime \prime}+B V^{\prime}+C V=0,
$$

where dashes denote the differentiation with respect to $z$, and

$$
V=[\hat{u}, \hat{w}, \hat{U}, \hat{W}]^{T}, T \text { stands for the tranpose, }
$$

$$
A=\left[\begin{array}{cccc}
a^{2} & 0 & 0 & 0 \\
0 & 1 & 0 & b^{2} \\
0 & 0 & 0 & 0 \\
0 & b^{2} & 0 & d^{2}
\end{array}\right],
$$




$$
\begin{aligned}
B & =-\left[\begin{array}{cccc}
0 & i q\left(1-a^{2}\right) & 0 & i q b^{2} \\
i q\left(1-a^{2}\right) & 0 & i q b^{2} & 0 \\
0 & i q b^{2} & 0 & i q d^{2} \\
i q b^{2} & 0 & i q d^{2} & 0
\end{array}\right], \\
C & =\left[\begin{array}{cccc}
-q^{2}-c_{11} & 0 & -b^{2} q^{2}-c_{12} & 0 \\
0 & -a^{2} q^{2}-c_{11} & 0 & -c_{12} \\
-b^{2} q^{2}-c_{12} & 0 & -d^{2} q^{2}-c_{22} & 0 \\
0 & -c_{12} & 0 & -c_{22}
\end{array}\right],
\end{aligned}
$$

where

$$
\begin{aligned}
& c_{11}=c^{2}\left(p^{2} R_{11}+p f^{2}\right), \quad c_{12}=c^{2}\left(p^{2} R_{12}-p f^{2}\right), \\
& c_{22}=c^{2}\left(p^{2} R_{22}+p f^{2}\right) .
\end{aligned}
$$

To solve the system of equations (11), we assume

$$
V(q, z, p)=X(q, p) e^{k z},
$$

and obtain the characteristic equation given by

$\operatorname{det}\left(k^{2} A+k B+C\right)=0$, which on simplification gives

$$
\begin{aligned}
& \left(k^{2} a^{2} c_{22}-q^{2} a^{2} c_{22}-c_{11} c_{22}+c_{12}^{2}\right)\left\{\left(d^{2}-b^{4}\right)\left(q^{2}-k^{2}\right)^{2}\right. \\
& +\left(c_{11} d^{2}+c_{22}-2 b^{2} c_{12}\right)\left(q^{2}-k^{2}\right) \\
& \left.+\left(c_{11} c_{22}-c_{12}^{2}\right)\right\}=0 .
\end{aligned}
$$

The solutions of equation (16) give the eigenvalues as

$$
k_{1}^{2}=\frac{A_{0}+\sqrt{A_{0}^{2}-4 B_{0}}}{2}, \quad k_{2}^{2}=\frac{A_{0}-\sqrt{A_{0}^{2}-4 B_{0}}}{2},
$$

$$
k_{3}^{2}=q^{2}+\frac{c_{11} c_{22}-c_{12}^{2}}{a^{2} c_{22}},
$$

where

$$
\begin{aligned}
& A_{0}=2 q^{2}+\frac{c_{11} d^{2}+c_{22}-2 b^{2} c_{12}}{d^{2}-b^{4}}, \\
& B_{0}=q^{4}+\frac{c_{11} d^{2}+c_{22}-2 b^{2} c_{12}}{d^{2}-b^{4}} q^{2}+\frac{c_{11} c_{22}-c_{12}^{2}}{d^{2}-b^{4}} .
\end{aligned}
$$

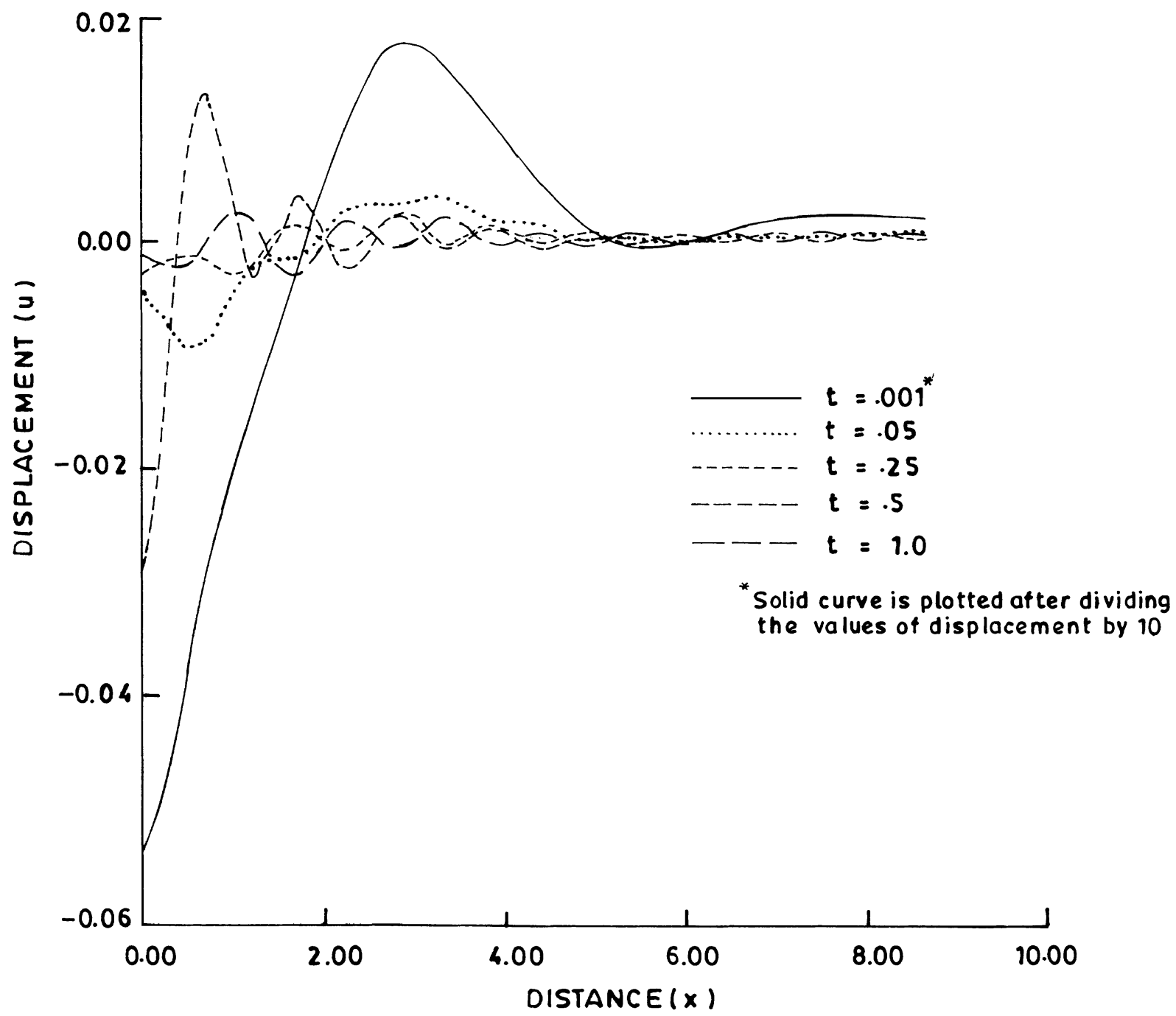

Figure 1. Tangential displacement distribution in the solid part $(\mathrm{u})$. 


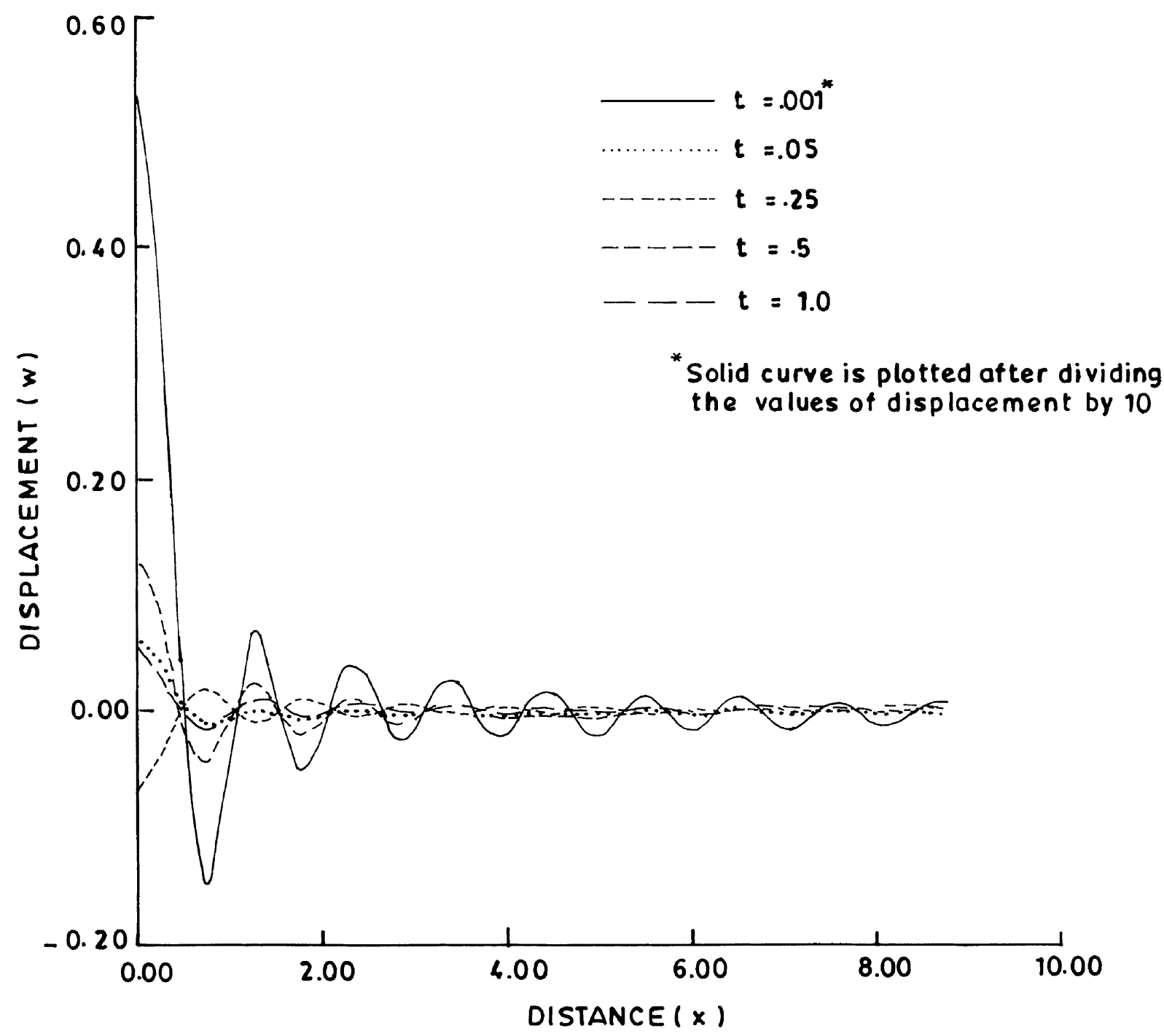

Figure 2. Normal displacement distribution in the solid part (w).

The eigenvectors $X(q, p)$ corresponding to different eigenvalues are obtained as

$$
\begin{aligned}
& X_{i}^{T}=\left[-q, k_{i},-q m_{i}, k_{i} m_{i}\right] \quad \text { and } \\
& X_{i+3}^{T}=\left[-q,-k_{i},-q m_{i},-k_{i} m_{i}\right], \quad(i=1,2,3),
\end{aligned}
$$

where

$$
\begin{array}{r}
m_{i}=\frac{c_{11}-\left(k_{i}^{2}-q^{2}\right)}{b^{2}\left(k_{i}^{2}-q^{2}\right)-c_{12}}=\frac{c_{12}-b^{2}\left(k_{i}^{2}-q^{2}\right)}{d^{2}\left(k_{i}^{2}-q^{2}\right)-c_{22}}, \\
(i=1,2,3) .
\end{array}
$$

Thus, the solution of equation (11) is given by

$$
V(q, z, p)=\sum_{i=1}^{3}\left\{B_{i} X_{i}(q, p) e^{k_{i} z}+B_{i+3} X_{i+3}(q, p) e^{-k_{i} z}\right\},
$$

where $B_{i}(i=1,2, \ldots, 6)$ are arbitary constants. The expression (21) represents the solution of the general plain strain problem for liquid-saturated porous iso- tropic elastic medium by applying the eigenvalue approach.

\subsection{Inversion of the transforms}

The transformed displacements and stresses are functions of depth $z$ and the parameters of Laplace and Fourier transforms $p$ and $q$, respectively, and hence are of the form $\hat{f}(q, z, p)$. To get the function $f(x, z, t)$ in the physical domain, first we invert the Fourier transform by using

$$
\begin{aligned}
\bar{f}(x, z, p) & =\int_{-\infty}^{\infty} e^{-i q x} \hat{f}(q, z, p) \mathrm{d} q \\
& =2 \int_{0}^{\infty}\left(\cos (q x) f_{e}-i \sin (q x) f_{0}\right) \mathrm{d} q,
\end{aligned}
$$

where $f_{e}$ and $f_{0}$ are even and odd parts of the function $\hat{f}(q, z, p)$, respectively. Thus, expression $(22)$ gives us the Laplace transform $\bar{f}(x, z, p)$ of the function $f(x, z, t)$.

Now, the function $f(x, z, p)$ in the expression (22) can be considered as the Laplace transform $\bar{g}(p)$ of 


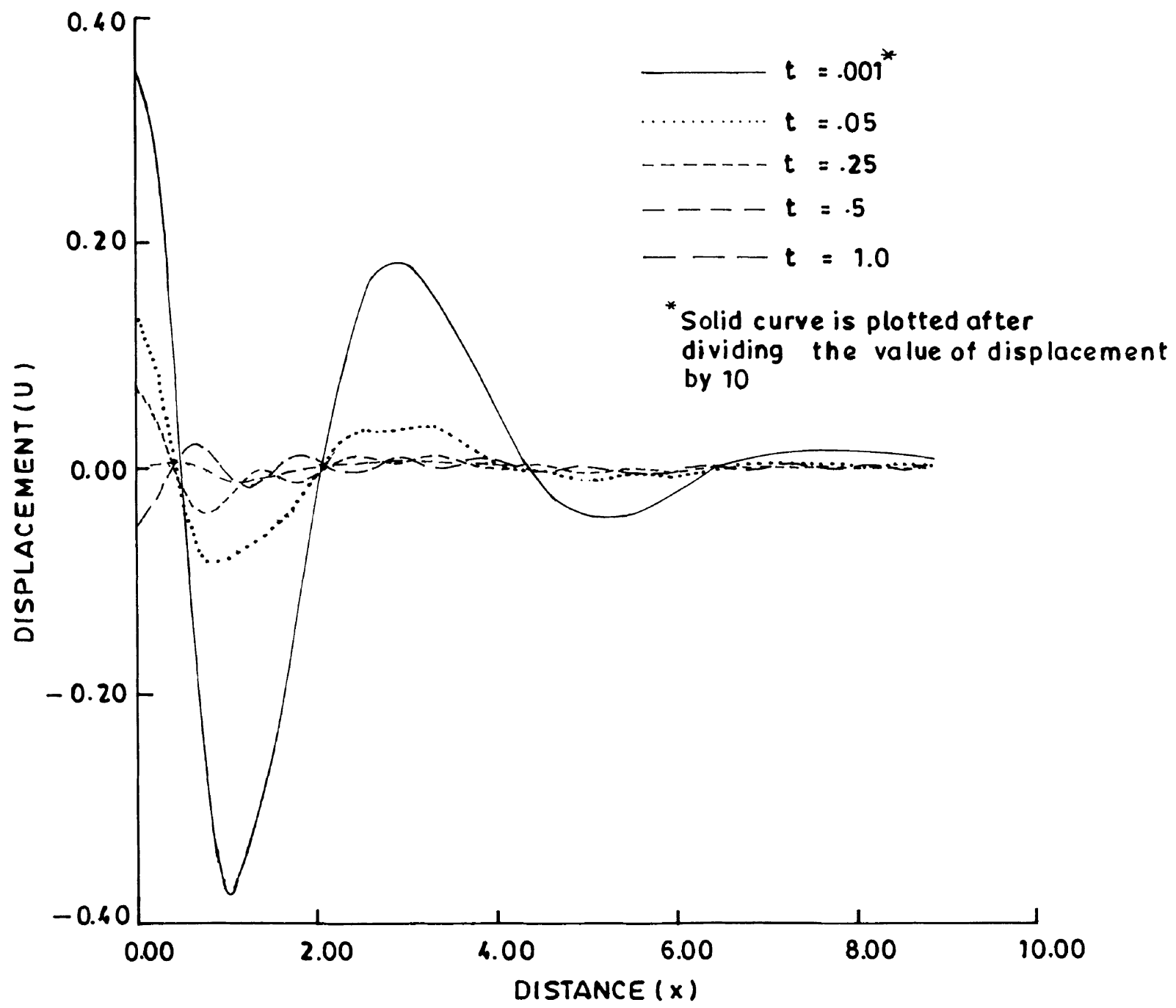

Figure 3. Tangential displacement distribution in the liquid part (U).

some function $g(t)$. Following Honing and Hirdes (1984), the Laplace transformed function $\bar{g}(p)$ can be inverted as given below.

The function $g(t)$ can be obtained by using

$$
g(t)=\frac{1}{2 \pi i} \int_{C-i \infty}^{C+i \infty} e^{p t} \bar{g}(p) d p,
$$

where $C$ is an arbitrary real number greater than all the real parts of the singularities of $\bar{g}(p)$. Taking $p=$ $C+i y$, we get

$$
g(t)=\frac{e^{C t}}{2 \pi} \int_{-\infty}^{\infty} e^{i t y} \bar{g}(C+i y) \mathrm{d} y .
$$

Now, taking $e^{-C t} g(t)$ as $h(t)$ and expanding it in the Fourier series form in $[0,2 \mathrm{~L}]$, we obtain the formula

$$
g(t)=g_{\infty}(t)+E_{D}
$$

where

$$
g_{\infty}(t)=\frac{C_{0}}{2}+\sum_{k=1}^{\infty} C_{k}, \quad 0 \leq t \leq 2 L,
$$

$$
C_{k}=\frac{e^{C t}}{L} R e\left[e^{i k \pi t / L} \bar{g}\left(C+\frac{i k \pi}{L}\right)\right],
$$

$E_{D}$ is the discretization error and can be made arbitrarily small by choosing $C$ large enough.

Since the infinite series in (26) can be summed up only to a finite number of $N$ terms, the approximate value of $g(t)$ becomes

$$
g_{N}(t)=\frac{C_{0}}{2}+\sum_{k=1}^{N} C_{k} \text { for } 0 \leq t \leq 2 L \text {. }
$$

Now, we introduce a truncation error $E_{T}$ that must be added to the discretization error to produce the total approximate error in evaluating $g(t)$ using the above formula. To accelerate the convergence, the discretization error and then the truncation error is reduced by using the 'korrecktur method' and the ' $\varepsilon$-algorithm', respectively, as given by Honig and Hirdes (1984).

The korrecktur method formula, to evaluate the function $g(t)$ is

$$
g(t)=g_{\infty}(t)-e^{-2 C L} g_{\infty}(2 L+t)+E_{D}^{\prime},
$$




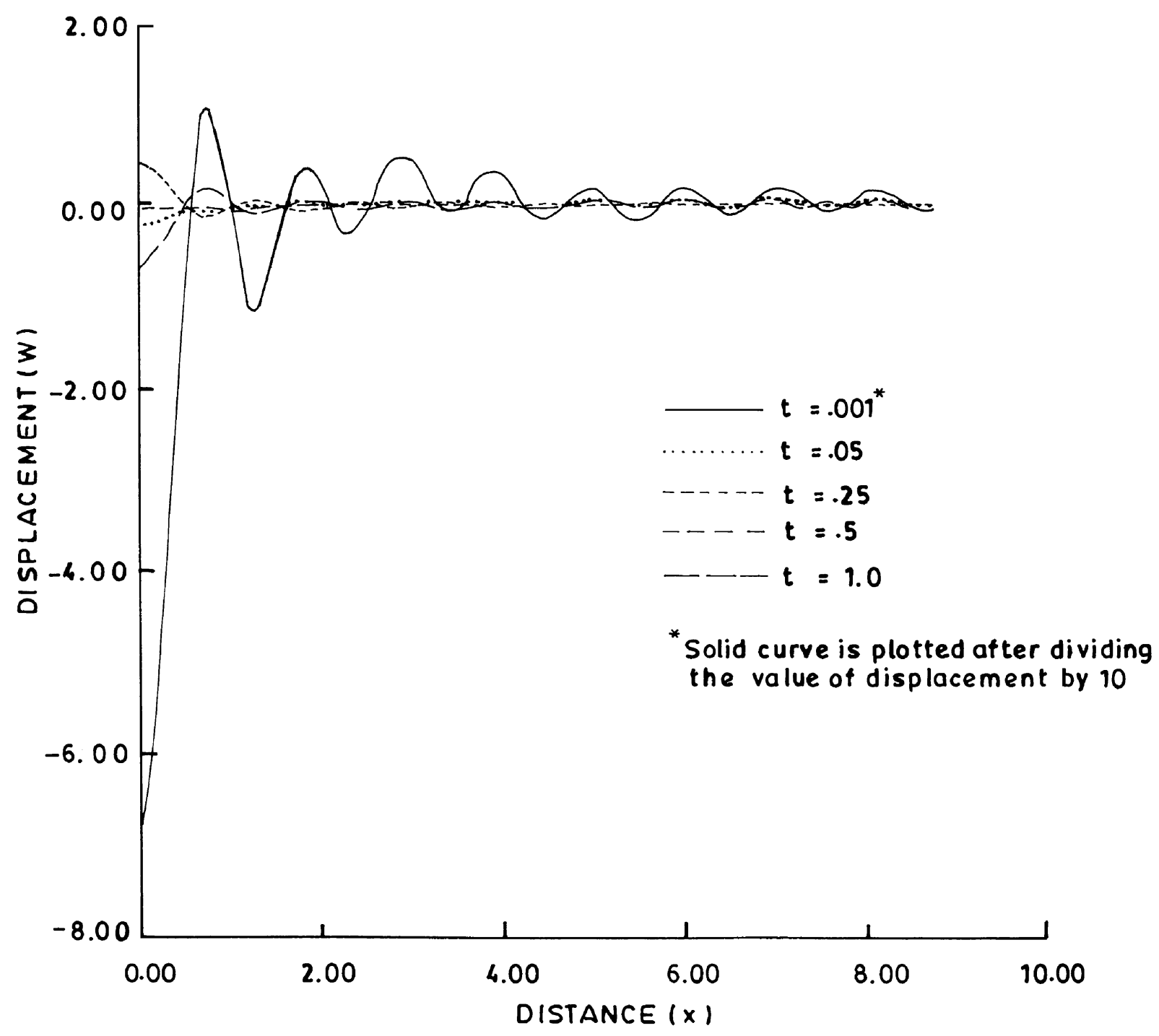

Figure 4. Normal displacement distribution in the liquid part (W).

where

$$
\left|E_{D^{\prime}}\right| \ll\left|E_{D}\right| \text {. }
$$

Thus, the approximate value of $g(t)$ becomes

$$
g_{N_{k}}(t)=g_{N}(t)-e^{-2 C L} g_{N^{\prime}}(2 L+t),
$$

where $N^{\prime}$ is an integer such that $N^{\prime}<N$.

We shall now describe the $\epsilon$-algorithm which is used to accelerate the convergence of the series in (27). Let $N$ be a natural number and $s_{m}=\sum_{k=1}^{m} C_{k}$ be the sequence of partial sums of equation (27). We define the $\epsilon$-sequence by

$$
\begin{aligned}
\epsilon_{0, m} & =0, \quad \epsilon_{1, m}=s_{m}, \\
\epsilon_{n+1, m} & =\epsilon_{n-1, m+1}+\frac{1}{\epsilon_{n, m+1}-\epsilon_{n, m}} ; \quad n, m=1,2,3,
\end{aligned}
$$

The sequence $\epsilon_{1,1}, \epsilon_{3,1}, \ldots, \epsilon_{N, 1}$ converges to $g(t)+$ $E_{D}-C_{0} / 2$ faster than the sequence of partial sums $S_{m}, m=1,2,3, \ldots$. The actual procedure to invert the
Laplace transform consists of equation (28) together with the $\epsilon$-algorithm. The values of $C$ and $L$ are chosen according to the criteria outlined by Honig and Hirdes (1984).

The last step is to calculate the integral in equation (22). The method for evaluating this integral is described by Press et al (1986), which involves the use of Romberg's integration with adaptive step size. This, also uses the results from successive refinements of the extended trapezoidal rule followed by extrapolation of the results to the limit when the step size tends to zero.

\subsection{Case: Concentrated point force}

Let us consider a concentrated force of magnitude $F=-F_{0} \delta(x) \delta(t)$ acting at the origin of the cartesian co-ordinate system in an infinite space, along the $z$ axis. The problem is plain strain with respect to $z$-axis and the boundary conditions on the plane $z=0$ are 


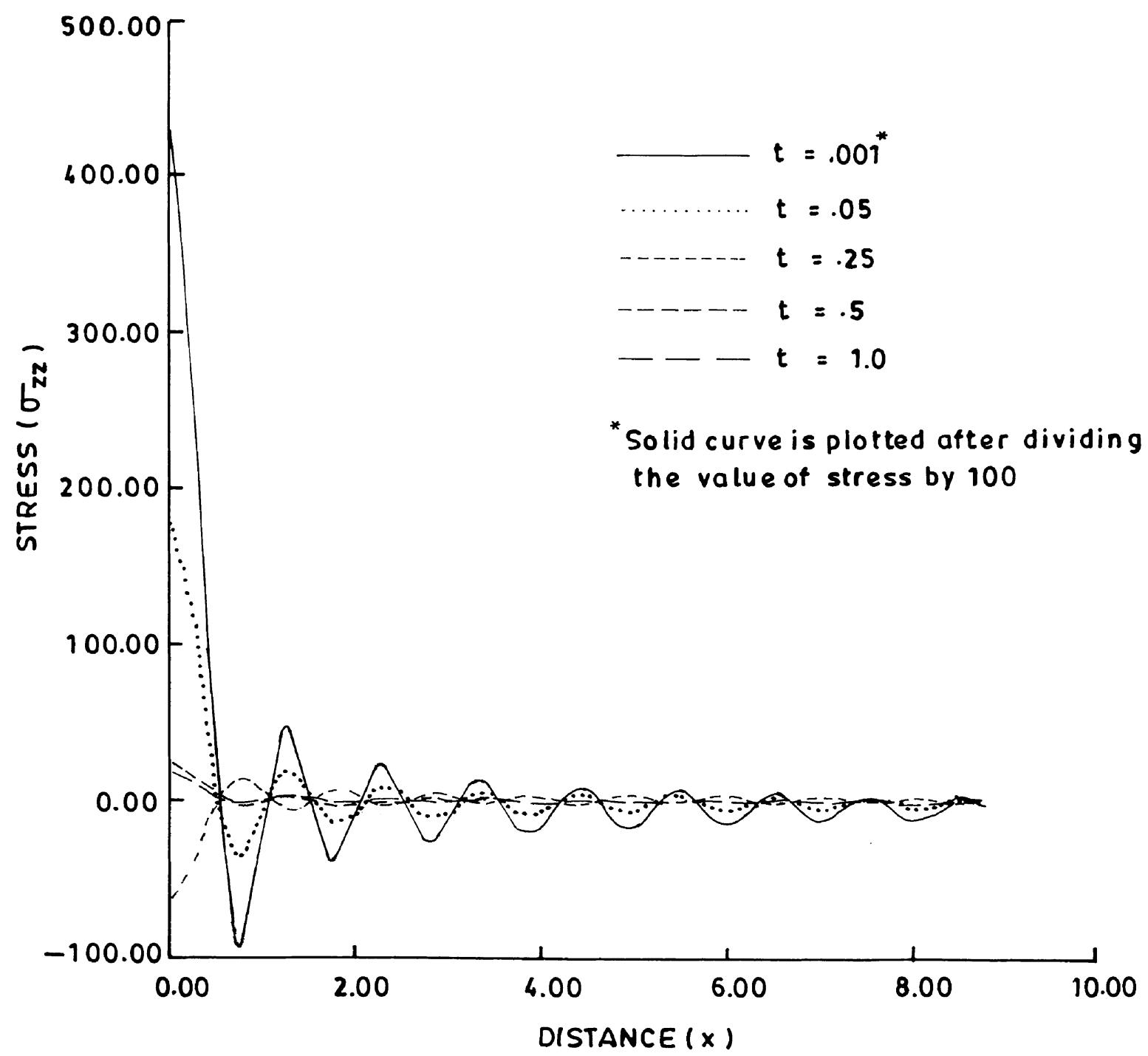

Figure 5. Normal stress distribution in the solid part $\left(\sigma_{z z}\right)$.

given as

$$
\begin{aligned}
& u\left(x, 0^{+}, t\right)-u\left(x, 0^{-}, t\right)=0, \\
& w\left(x, 0^{+}, t\right)-w\left(x, 0^{-}, t\right)=0, \\
& W\left(x, 0^{+}, t\right)-W\left(x, 0^{-}, t\right)=0, \\
& \sigma_{z z}\left(x, 0^{+}, t\right)-\sigma_{z z}\left(x, 0^{-}, t\right)=-F_{0} \delta(x) \delta(t), \\
& \sigma_{x z}\left(x, 0^{+}, t\right)-\sigma_{x z}\left(x, 0^{-}, t\right)=0, \\
& \sigma\left(x, 0^{+}, t\right)-\sigma\left(x, 0^{-}, t\right)=F_{0} \delta(x) \delta(t) .
\end{aligned}
$$

After non-dimensionalizing equation (29) by using (6) and $F_{0}^{\prime}=F_{0} / P$, and suppressing the dashes and applying the Laplace and then the Fourier transformation as defined by (9) and (10), we obtain

$$
\begin{aligned}
& \hat{u}\left(q, 0^{+}, p\right)-\hat{u}\left(q, 0^{-}, p\right)=0, \\
& \hat{w}\left(q, 0^{+}, p\right)-\hat{w}\left(q, 0^{-}, p\right)=0, \\
& \hat{W}\left(q, 0^{+}, p\right)-\hat{W}\left(q, 0^{-}, p\right)=0, \\
& \hat{\sigma}_{z z}\left(q, 0^{+}, p\right)-\hat{\sigma}_{z z}\left(q, 0^{-}, p\right)=-\frac{F_{0}}{2 \pi},
\end{aligned}
$$

$$
\begin{aligned}
& \hat{\sigma}_{x z}\left(q, 0^{+}, p\right)-\hat{\sigma}_{x z}\left(q, 0^{-}, p\right)=0, \\
& \hat{\sigma}\left(q, 0^{+}, p\right)-\hat{\sigma}\left(q, 0^{-}, p\right)=\frac{F_{0}}{2 \pi} .
\end{aligned}
$$

Now, from (21) and (3), the transformed displacements and stresses are given by

for $z \geq \mathbf{0}$

$$
\begin{aligned}
\hat{u}(q, z, p)= & -q\left(B_{4} e^{-k_{1} z}+B_{5} e^{-k_{2} z}+B_{6} e^{-k_{3} z}\right), \\
\hat{w}(q, z, p)= & -\left(k_{1} B_{4} e^{-k_{1} z}+k_{2} B_{5} e^{-k_{2} z}+k_{3} B_{6} e^{-k_{3} z}\right), \\
\hat{U}(q, z, p)= & -q\left(m_{1} B_{4} e^{-k_{1} z}+m_{2} B_{5} e^{-k_{2} z}\right. \\
& \left.+m_{3} B_{6} e^{-k_{3} z}\right) \\
\hat{W}(q, z, p)= & -\left(k_{1} m_{1} B_{4} e^{-k_{1} z}+k_{2} m_{2} B_{5} e^{-k_{2} z}\right. \\
& \left.+k_{3} m_{3} B_{6} e^{-k_{3} z}\right), \\
\hat{\sigma}_{z z}(q, z, p)= & \frac{H}{\rho \alpha_{2}^{2}}\left(H_{1} B_{4} e^{-k_{1} z}+H_{2} B_{5} e^{-k_{2} z}\right. \\
& \left.+H_{3} B_{6} e^{-k_{3} z}\right),
\end{aligned}
$$




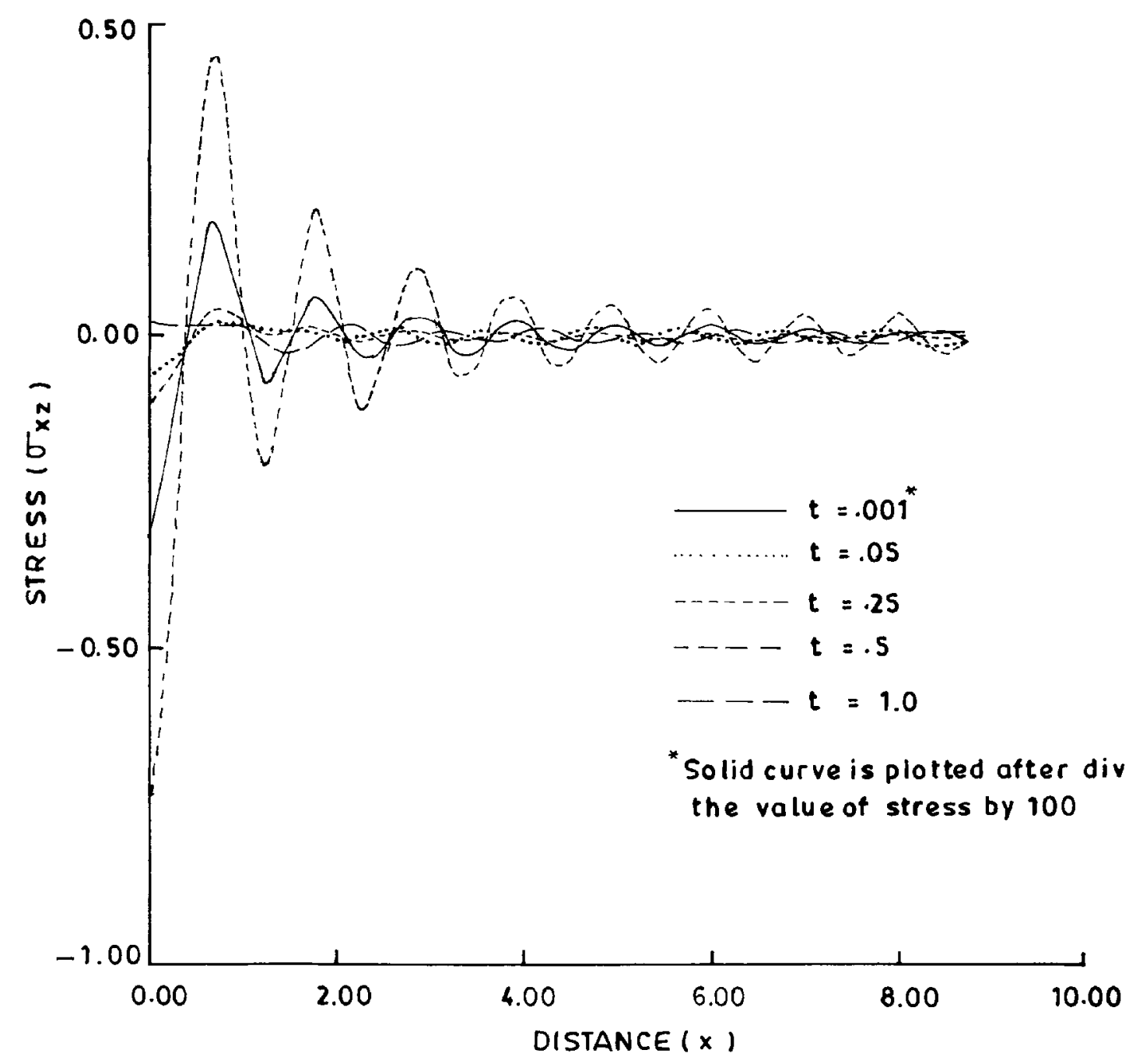

Figure 6. Tangential stress distribution in the solid part $\left(\sigma_{x z}\right)$.

$$
\begin{gathered}
\hat{\sigma}_{x z}(q, z, p)=\frac{H}{\rho \alpha_{2}^{2}} a^{2} q(1+i)\left(k_{1} B_{4} e^{-k_{1} z}+k_{2} B_{5} e^{-k_{2} z}\right. \\
\left.\quad+k_{3} B_{6} e^{-k_{3} z}\right) \\
\hat{\sigma}(q, z, p)=\frac{H}{\rho \alpha_{2}^{2}}\left(J_{1} B_{4} e^{-k_{1} z}+J_{2} B_{5} e^{-k_{2} z}\right. \\
\left.+J_{3} B_{6} e^{-k_{3} z}\right) .
\end{gathered}
$$

for $z \leq \mathbf{0}$

$$
\begin{aligned}
\hat{u}(q, z, p)= & -q\left(B_{1} e^{k_{1} z}+B_{2} e^{k_{2} z}+B_{3} e^{k_{3} z}\right) \\
\hat{w}(q, z, p)= & \left(k_{1} B_{1} e^{k_{1} z}+k_{2} B_{2} e^{k_{2} z}+k_{3} B_{3} e^{k_{3} z}\right), \\
\hat{U}(q, z, p)= & -q\left(m_{1} B_{1} e^{k_{1} z}+m_{2} B_{2} e^{k_{2} z}+m_{3} B_{3} e^{k_{3} z}\right), \\
\hat{W}(q, z, p)= & \left(k_{1} m_{1} B_{1} e^{k_{1} z}+k_{2} m_{2} B_{2} e^{k_{2} z}\right. \\
& \left.+k_{3} m_{3} B_{3} e^{k_{3} z}\right) \\
\hat{\sigma}_{z z}(q, z, p)= & \frac{H}{\rho \alpha_{2}^{2}}\left(H_{1} B_{1} e^{k_{1} z}+H_{2} B_{2} e^{k_{2} z} H_{3} B_{3} e^{k_{3} z}\right), \\
\hat{\sigma}_{x z}(q, z, p)= & -\frac{H}{\rho \alpha_{2}^{2}} a^{2} q(1+i)\left(k_{1} B_{1} e^{k_{1} z}\right. \\
& \left.+k_{2} B_{2} e^{k_{2} z}+k_{3} B_{3} e^{k_{3} z}\right)
\end{aligned}
$$$$
\hat{\sigma}(q, z, p)=\frac{H}{\rho \sigma_{2}^{2}}\left(J_{1} B_{1} e^{k_{1} z}+J_{2} B_{2} e^{k_{2} z}+J_{3} B_{3} e^{k_{3} z}\right),
$$

where

$$
\begin{gathered}
H_{j}=i q^{2}\left\{\left(1-2 a^{2}\right)+b^{2} m_{j}\right\}+\left(1+b^{2} m_{j}\right) k_{j}^{2}, \\
J_{j}=\left(b^{2}+d^{2} m_{j}^{2}\right)\left(k_{j}^{2}+i q^{2}\right), \quad(j=1,2,3),
\end{gathered}
$$

and 'i' stands for iota, i.e., imaginary.

Making use of (31) and (32) in the transformed conditions (30), we obtain a system of six equations in six unknowns $B_{1}, B_{2}, B_{3}, B_{4}, B_{5}, B_{6}$, which on solving gives

$$
\begin{aligned}
& B_{1}=-B_{4}=\frac{\left(H_{3}-H_{2}\right)+\left(J_{3}-J_{2}\right)}{4 \pi \Delta} F_{0}\left(\frac{\rho \alpha_{2}^{2}}{H}\right), \\
& B_{2}=-B_{5}=\frac{\left(H_{3}-H_{1}\right)+\left(J_{3}-J_{1}\right)}{4 \pi \Delta} F_{0}\left(\frac{\rho \alpha_{2}^{2}}{H}\right), \\
& B_{3}=-B_{6}=\frac{\left(H_{2}-H_{1}\right)+\left(J_{2}-J_{1}\right)}{4 \pi \Delta} F_{0}\left(\frac{\rho \alpha_{2}^{2}}{H}\right),
\end{aligned}
$$


where

$$
\Delta=\left(J_{2}-J_{1}\right)\left(H_{3}-H_{1}\right)-\left(H_{2}-H_{1}\right)\left(J_{3}-J_{1}\right) .
$$

Thus, the equations (31) and (32) give the displacements and stresses in the transformed domain, which on inversion, provide the displacements and stresses in the physical domain.

\section{Numerical results and discussion}

The displacements and stresses in the solid and liquid parts of the porous aggregate have been computed, separately. For the purpose of numerical calculations, the medium considered is of sandstone saturated with kerosene. Following Yew and Jogi (1976), the numerical values for various non-dimensional parameters are taken as

$$
\begin{aligned}
a & =0.5267, \quad b=0.2731, \quad c=0.5029, \quad d=0.1809 \\
R_{11} & =0.90124, \quad R_{12}=-0.00099, \quad R_{22}=0.10076
\end{aligned}
$$

Also, we have taken

$$
\frac{\rho \alpha_{2}^{2}}{H}=0.21398, \quad \frac{b}{\rho \omega}=1.0, \quad F_{0}^{\prime}=\frac{F_{0}}{p}=1.0 .
$$

Using the numerical technique described above to invert the transforms in equations (31) and (32), the displacement and stress components are calculated against the distance ' $x$ ' for the following values of time $t=0.001,0.05,0.25,0.5$ and 1.0 at the plane $z=1.0$. The results, thus obtained, are shown in figures 1-7. The displacements and stresses for the value $t=0.001$ are very large in comparison to the values at larger ' $t$ '. Thus, to show the curves for all the values of ' $t$ ' in the same figure, for each case of displacement and stress components, we plot the displacement curves and stress curves corresponding to time $t=0.001$, after dividing the values by 10 and 100, respectively. The curves corresponding to this value of $t$, i.e., $t=0.001$ are shown by solid curves in the figures. It is observed that the maximum (absolute) displacement and stress

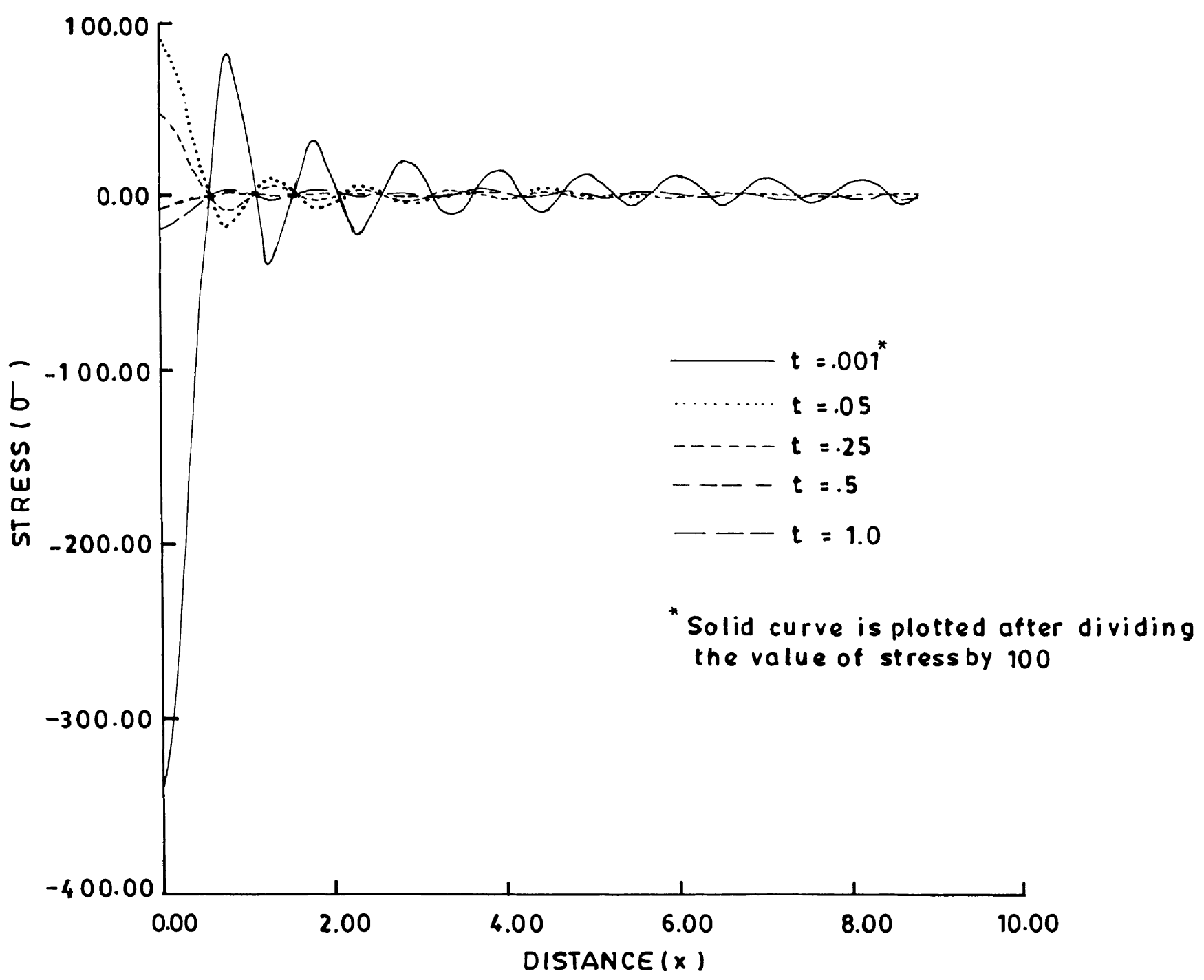

Figure 7. Normal stress distribution in the liquid part $(\sigma)$. 
occur at the minimum time. i.e., $t=0.001$, in response to the impulsive force. Further, with the increase in time, for a fixed position of ' $x$ ', the displacements and stresses follow oscillatory pattern with reference to time, with maximum (absolute) displacement and stress at the initial value, i.e., $x=0$. Ultimately, they die out with the further increase in time. Also, it is observed that with the increase in the distance, i.e., value of ' $x$ ', away from the source, they become zero in all the cases. That means, we can say that all these quantities are maximum (absolute) at minimum time, i.e., $t=0.001$ and distance, i.e., $x=0$ and ultimately become zero with the passage of time as well as with the increase in distance from the source. The magnitude of displacements and stresses are much higher in the case of normal displacement and stress components $\left(w\right.$ and $\left.\sigma_{z z}\right)$ in comparison with the tangential displacement and stress components $\left(u\right.$ and $\left.\sigma_{x z}\right)$ in the solid part of the medium. Also, in the liquid part, the magnitude of normal displacement $(W)$ are higher in comparison with the tangential displacement $(U)$. However, the displacement components in the liquid constituents have higher magnitudes in comparison to their respective solid constituents.

As the medium considered is liquid-saturated porous, which is a two phase medium involving an elastic solid matrix with pores saturated with fluid, the displacement travelling through these different constituents of the medium, suffers sudden changes, resulting in inconsistent pattern. Otherwise, when it passes through either solid or liquid, it shows a con- sistent pattern. That's why the patterns are different in different cases.

Assuming the earthquake preparation region as liquid-saturated porous medium, displacements and stresses due to an active source can be computed from time to time by using this eigenvalue approach.

\section{Acknowledgement}

One of the authors (N R Garg) is thankful to U.G.C. for supporting a minor research project.

\section{References}

Biot M A. 1956a General Solution of the equations of elasticity and consolidation for a porous meterial; J. Appl. Mech. 23 91-95

Biot M A 1956b The theory of propagation of elastic waves in a fluid-saturated porous solid; J. Acoust. Soc. Am. 28 168-191

Cramplin S 1987 The basis for earthquake prediction; Geophys. J. Roy. Astro. Soc. 91, 331-347

Deresiewicz H and Skalak R. 1963 On uniqueness in dynamic poro-elasticity; Bull. Seism. Soc. Am. 53 783-789

Honig G and Hirdes U 1984 A method for the numerical inversion of the Laplace transform; J. Comp. Appl. Math. 10 113-132

Press W H, Teukolsky S A, Vellerling W T and Flannery B P 1986 Numerical Recipes (Cambridge: Cambridge University Press)

Yew C H and Jogi P N 1976 Study of wave motions in fluidsaturated porous rocks; J. Acoust. Soc. Am. $602-8$ 